\title{
Noncanonical cytoplasmic processing of viral microRNAs
}

\author{
JILLIAN S. SHAPIRO, ${ }^{1,2}$ ANDREW VARBLE, ${ }^{1,2}$ ALISSA M. PHAM, ${ }^{1,2}$ and BENJAMIN R. TENOEVER ${ }^{1,2,3}$ \\ ${ }^{1}$ Microbiology Graduate School Training Program, Mount Sinai School of Medicine, New York, New York 10029, USA \\ ${ }^{2}$ Department of Microbiology, Mount Sinai School of Medicine, New York, New York 10029, USA \\ ${ }^{3}$ Global Health and Emerging Pathogens Institute, Mount Sinai School of Medicine, New York, New York 10029, USA
}

\begin{abstract}
Cellular utilization of RNA interference (RNAi) as a mechanism to combat virus infection is thought to be restricted to plants and invertebrates. In vertebrates, antiviral defenses are largely dependent on interferons (IFNs), with the use of small RNAs restricted to microRNA (miRNA)-mediated targeting of host transcripts. Here we demonstrate that incorporation of a primary miRNA into a cytoplasmic virus results in the formation of a Dicer-dependent, DGCR8-independent, mature miRNA capable of conferring RNAi-like activity. Processing of the viral mirtron-like product (virtron) is indistinguishable from endogenous miRNA maturation and elicits post-transcriptional gene silencing, albeit at a reduced level. Furthermore, virtrons impose Dicerdependent, microprocessor-independent, and IFN-independent interference on virus replication in a sequence-specific manner. Taken together, these results suggest the existence of a noncanonical, small-RNA-based activity capable of processing cytoplasmic hairpins and perhaps contributing to the cell's antiviral arsenal.
\end{abstract}

Keywords: synthetic biology; RNA interference; viral engineering; siRNA delivery; antiviral vector

\section{INTRODUCTION}

The generation of small RNAs is a pivotal intracellular defense against pathogens in a broad range of plants and a subset of invertebrates (Hutvagner and Zamore 2002; Reinhart et al. 2002; Umbach and Cullen 2009). Generally referred to as small interfering RNAs (siRNAs), this class of RNA interference (RNAi) is generated by cytoplasmic RNA-dependent RNA polymerases (RdRp) that copy back long precursor double-stranded RNAs (dsRNAs) derived from the pathogen (Zamore et al. 2000). This RdRpdependent dsRNA is then further modified to short RNAs of 20-25 nt in length through the processive activity of Dicer and used to mediate cleavage of the pathogen RNA from which it derived (Zamore et al. 2000).

Despite the conservation of many components involved in the synthesis of siRNAs, the biological activity of small RNAs in vertebrates appears largely limited to the generation of microRNAs (miRNAs) (Ambros 2004). Unlike siRNAs, miRNAs are processed directly from germline encoded transcripts generated predominantly from RNA polymerase II-derived noncoding or intronic RNA (Bartel

Reprint requests to: Benjamin R. tenOever, Microbiology Graduate School Training Program, Mount Sinai School of Medicine, New York, NY 10029, USA; e-mail: benjamin.tenoever@mssm.edu; fax: (212) 534-1684.

Article published online ahead of print. Article and publication date are at http://www.rnajournal.org/cgi/doi/10.1261/rna.2303610.
2004). For canonical miRNAs, initial processing of the primary miRNA (pri-miRNA) depends on the microprocessor, which consists of the RNAse III enzyme Drosha and the dsRNA-binding protein DGCR8 (Lee et al. 2003; Wang et al. 2008). The microprocessor yields a 60-70-nt premiRNA, which is exported from the nucleus in an Exportin-5-dependent manner (Yi et al. 2003) and is further modified by Dicer, the same endoribonuclease enzyme used to generate siRNAs in other organisms (Bernstein et al. 2001; Billy et al. 2001; Ketting et al. 2001; Knight and Bass 2001). Dicer is thought to recognize the 2-nt 3'-overhang of the pre-miRNA and make a single cleavage, $\sim 20 \mathrm{nt}$ from the base of the stem, thereby generating a dsRNA duplex (MacRae et al. 2007).

In addition to the canonical generation of pre-miRNAs, a very small subset of miRNAs, referred to as mirtrons, do not require the microprocessor as these are generated from very short introns by the spliceosome, are debranched, and then refold to form pre-miRNAs (Berezikov et al. 2007; Ruby et al. 2007). The pre-miRNA products are then exported to the cytoplasm in an Exportin-5-dependent manner (Okamura et al. 2007; Ruby et al. 2007). Mirtrons, like canonical pre-miRNAs, are Dicer-dependent but are DGCR8, and therefore microprocessor, independent (Berezikov et al. 2007; Ruby et al. 2007).

Regardless of the source of the pre-miRNA, the Dicerdependent duplex, now containing $3^{\prime}$-overhangs at both 
ends, is loaded into the RNA-induced silencing complex (RISC), where the thermal stability of each $5^{\prime}$ end determines which strand is used as the guide strand and which one is discarded as the passenger or microRNA star strand (Khvorova et al. 2003; Schwarz et al. 2003; Wang et al. 2009). RISC, loaded with a mature miRNA, then binds to target messenger RNAs (mRNAs) through a 5 '-seed sequence generally encompassing bases 2-8 (Bartel 2009). Binding of the seed sequence to the $3^{\prime}$-untranslated regions (UTRs) of mRNAs results in both translational repression and RNA destabilization through Glycinetryptophan protein of $182 \mathrm{kDa}$ (GW182)-mediated deadenylation (Zipprich et al. 2009). While miRNA binding to the $3^{\prime}$-UTR is the most common form of posttranscriptional gene silencing (PTGS), the open reading frame (ORF) can also be targeted (Chi et al. 2009). The PTGS activity of miRNAs is thought to fine-tune global transcription of a wide range of mRNAs, modulating each one no more than twofold to threefold (Selbach et al. 2008).

Despite the presence of small RNAs in vertebrates, lack of an endogenous cytoplasmic RdRp prevents the production of bona fide siRNAs. Instead, vertebrates have evolved distinct pathogen recognition receptors (PRRs) to defend against viral replication. PRRs recognize distinct classes of viral replication intermediates, which, similar to invertebrate defenses, include the formation of foreign RNA nucleic acid structures (Iwasaki and Medzhitov 2010). Detection of virus infection results in the synthesis of Type I interferons (IFN-I) and the up-regulation of more than 100 IFN-stimulated genes that confer viral protection (Garcia-Sastre and Biron 2006). The emergence of IFNI-dependent antiviral activity has appeared to replace endogenous siRNA defenses some time during vertebrate evolution. However, even with this apparent loss of the intracellular siRNA utility, incorporating perfectly complementary artificial endogenous miRNA targets into viruses has been found to effectively inhibit replication in a similar manner to siRNAs, suggesting that perhaps vestigial aspects of this antiviral pathway still exist (tenOever 2009). Evidence of cross-talk between PRR- and RNAi-mediated antiviral defenses has also been directly demonstrated, as IFN-I can induce miRNA expression, altering both the cellular transcriptome and directly targeting virus replication (O'Connell et al. 2007; Pedersen et al. 2007). In addition, recent deep sequencing efforts have identified a number of small RNAs from cytoplasmic viruses, suggesting that the cell may be able to directly cleave viral genomic segments (Parameswaran et al. 2010). To ascertain whether viral dsRNA still has the capacity to be processed by Dicer and used in an antiviral capacity in vertebrate cells, we engineered Sindbis virus to encode a pri-miRNA, thereby relieving the necessity for $\mathrm{RdRp}$ to produce dsRNA and generating both substrate and target for mediating antiviral activity.

\section{RESULTS AND DISCUSSION}

\section{Sindbis virus-derived subgenomic pri-miR-124-2 induces cytoplasmic processing of miR-124}

In an effort to determine the consequences of grafting a primiRNA locus into a virus that replicates exclusively in the cytoplasm, we inserted the mmu-miR-124-2 pri-miRNA locus into an extra, nonessential subgenomic fragment of Sindbis virus (Fig. 1A; Cheng et al. 1996). miR-124 is
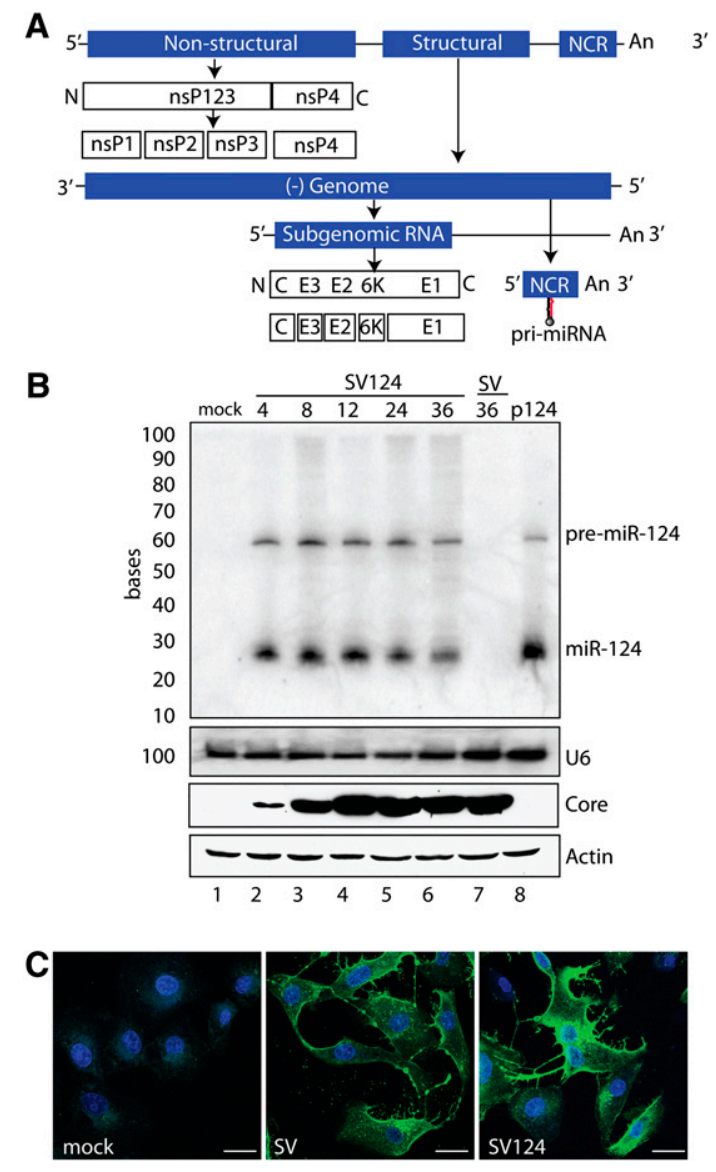

FIGURE 1. (A) Schematic representation of Sindbis viral products. $5^{\prime}$ and $3^{\prime}$ ends of mRNA and negative-strand genome products are depicted as are $\mathrm{NH} 2(\mathrm{~N})$ and $\mathrm{COOH}(\mathrm{C})$ terminals of polyproteins. The noncoding region (NCR) represents the extra subgenomic insertion site where the pri-miRNA transcript was incorporated. The nonstructural genes (nsP1-4) are translated into a large polyprotein that forms four unique nonstructural proteins. The complementary minus strand $[(-)$ Genome] is used as a template for the genomic RNA along with both the subgenomic mRNA and the extra subgenomic NCR depicted. The endogenous subgenomic message is translated into a second polyprotein that is processed into the $\mathrm{C}, \mathrm{E} 3, \mathrm{E} 2,6 \mathrm{~K}$, and $\mathrm{E} 1$ proteins. $(B)$ Human fibroblasts mock-treated, transfected with miR-124 producing plasmid (p124), or infected with SV or SV124 (MOI of 5) and harvested at the indicated hours post-infection (hpi). (Upper two frames) Northern blots probed for miR-124 (top) and U6 (bottom). (Lower two frames) Immunoblots depicting Sindbis virus core protein and actin. (C) Confocal microscopy of cells mock-treated or infected with SV or SV124 (MOI of 2). Cells stained for Sindbis virus core protein (green) and cell nuclei (blue). Scale bar, $10 \mu \mathrm{m}$. 
produced through canonical processing (Babiarz et al. 2008) and is endogenously expressed to high levels in neuronal tissue (Makeyev et al. 2007). In addition, artificial production of miR-124 has been demonstrated when cloned as an intron of a plasmid or when inserted into the genome of influenza A virus (Varble et al. 2010).

Insertion of the putative RNA hairpin did not prevent generation of recombinant virus by plasmid rescue as both control Sindbis (SV) and the miR-124-2 encoding virus (SV124) propagated to stock titers of $\sim 1 \times 10^{7} \mathrm{pfu} / \mathrm{mL}$ plaque forming units (pfus) per milliliter (data not shown). To ascertain whether SV124 produced any small RNAs derived from the putative stem-loop, we infected fibroblasts at a high multiplicity of infection (MOI of 5 ) for 4,8 , 12, 24, and $36 \mathrm{~h}$ (Fig. 1B). RNAs of $<150 \mathrm{nt}$ were subsequently analyzed by small RNA Northern blot (Pall and Hamilton 2008) and compared to mock treatment, SV infection, and the transfection of a miR-124-producing plasmid (p124). Surprisingly, SV124 produced abundant levels of both a 60 -nt pre-miRNA and an $\sim 20$-nt mature miR-124. The levels of both the pre-miR-124 and miR-124 were comparable to that observed from plasmid-based overexpression as early as $4 \mathrm{~h}$ post-infection (hpi) and were maintained for the duration of the infection. Furthermore, the levels of Sindbis core protein were comparable between SV and SV124, suggesting no obvious replicative defects in human fibroblasts. In addition, confocal microscopy demonstrated robust cytoplasmic replication of both SV and SV124 with near 100\% cell infectivity (Fig. 1C).

The comparable levels of core protein and cell infectivity strongly suggest that the addition of the pri-miR-124 locus has minimal impact on the overall viral life cycle. This result is surprising given that the predicted formation of the $\sim 50$-nt stem-loop could potentially result in steric hindrance on the viral RdRp, processing of the hairpin, and cleavage of genomic material, and/or that the dsRNA motif formed by the virus could serve as a pathogen-associated molecular pattern (PAMP). Based on the comparable properties of the viral recombinants, it appears that a viral protein prevents the formation of the genomic hairpin and/or that the viral RdRp can dissolve the structure during replication. Furthermore, similar levels of core protein suggest miRNAmediated self-targeting does not occur in the context of immortalized human fibroblasts.

\section{SV124 produces Dicer-dependent, Exportin-5-independent, cytoplasmic miR-124 and miR-124-like small RNAs}

Given the cytoplasmic localization of SV124, the appearance of an $\sim 60$-nt pre-miRNA (Fig. 1B) suggests that either the Sindbis-derived subgenomic pri-miR-124 translocates to the nucleus, where it is trimmed by the microprocessor, or that a noncanonical cytoplasmic-based, microprocessorindependent mechanism is responsible for Sindbis-generated
miRNAs. To ascertain whether Sindbis-derived miR-124 undergoes canonical miRNA processing, we infected wildtype murine fibroblasts and compared the RNA from these infections to identical treatments performed in fibroblasts lacking Dicer (Fig. 2A). Following 24 h of infection, wildtype murine fibroblasts demonstrated robust synthesis of the neuronal-specific miR-124 from SV124 infections. Sindbis-derived miR-124 was dependent on Dicer activity as cells not expressing the endoribonuclease, as a result of disruption in the Dicer gene (Dcr1), demonstrated a complete loss of both SV-derived miR-124, as well as endogenous miR-93.

We next determined whether Exportin-5 was required for Sindbis-mediated miR-124 production. As Exportin-5 has been found to be essential for the nuclear export of all endogenous miRNAs (Yi et al. 2003), we reasoned that, if the Sindbis-derived miR-124 mRNA was processed in the nucleus, it would be reduced or eliminated by the knockdown of Exportin-5. To this end, we treated human fibroblasts with scrambled RNAi $(\mathrm{Scbl})$ or a pool of RNAi targeting the transcript of the Exportin-5 gene (Xpo5) (Fig. 2B). Forty-eight hours post-transfection (hpt), we found that Exportin-5 siRNA pools effectively eliminated Exportin-5 protein expression and subsequently infected cells with SV or SV124. Twenty-four hours post-infection, total RNA was
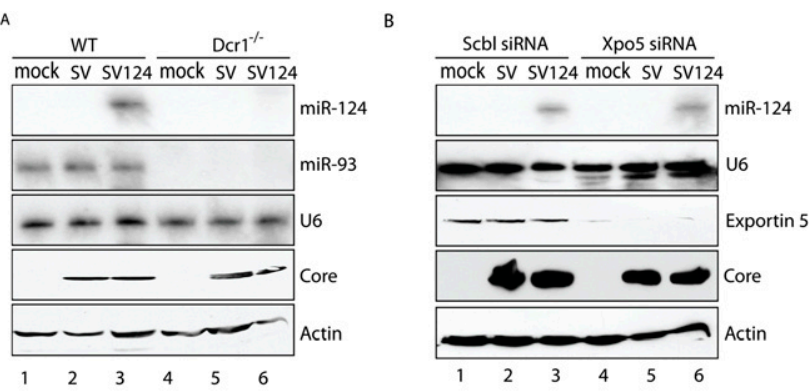

\section{C}

CUCUGCUCUCCGUGUUCACAGCGGACCUUGAUUUAAUGUCAUACAAUUAAGGCACGCGGUGAUUGCCAAGAGCGGAG

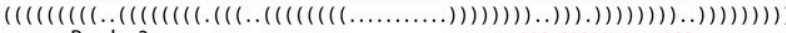
...... Reads: 3 Reads: 1 ............................. Reads: 34 Reads: 19

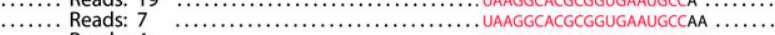
$\ldots \ldots$ Reads: 4 ....................................... UAAGGGAACGCGGUGAAUGCCAAG........

FIGURE 2. (A) Murine embryonic fibroblasts derived from wild-type (WT) or Dicer-deficient $\left(D c r 1^{-/}\right)$mice mock-treated or infected with SV or SV124 for $24 \mathrm{~h}$ (MOI of 1). (Top three panels) Northern blots probed for miR-124 (top), miR-93 (middle), and U6 (bottom). (Bottom two panels) Western blots for Sindbis virus core protein and actin. (B) Human fibroblasts transfected with scrambled short interfering RNAs (Scbl siRNA) or siRNAs directed against Exportin-5 (Xpo5 siRNA). Forty-eight hours post-transfection, cells were mocktreated or infected with SV or SV124 for $24 \mathrm{~h}$ (MOI of 1). (Top two panels) Northern blot probed for miR-124 (top) and U6 (below). (Bottom three panels) Immunoblots for Exportin-5, Sindbis core, and actin. $(C)$ Sequence analysis of Sindbis-derived miR-124. The premiR-124 sequence is depicted at the top, with the mature miR-124 sequence in red and the predicted secondary structure below. The number of reads corresponding to each RNA species is indicated. 
collected, and subsequent small RNA Northern blot revealed no change in Sindbis-derived levels of miR-124, suggesting that the miR-124 was not of nuclear origin.

To better define the characteristics of the noncanonical Sindbis-generated cytoplasmic miR-124, we analyzed the small RNA pool from cells infected with SV124 (Fig. 2C). Small RNAs between 17 and $25 \mathrm{nt}$ were isolated and ligated with $5^{\prime}$ and $3^{\prime}$ linkers sequentially. Linker-specific reverse transcription was performed, and subsequent PCR amplification was executed with 5' -pre-miR-124- and $3^{\prime}$-linkerspecific primers or vice versa, in an effort to sample the miR-124 and miR-124-like products without bias. Analysis of more than 50 individual clones demonstrated that while $50 \%$ of the Sindbis-derived miR-124 sequences matched perfectly to endogenous miR-124, 44\% demonstrated some degree of $3^{\prime}$ heterogeneity.

Taken together, the dependence on Dicer, the high percentage of aberrant $3^{\prime}$ processing, and the independence on Exportin-5 suggest that Sindbis-derived miR-124 represents an uncharacterized cellular pathway capable of processing hairpin RNA.

\section{Sindbis-derived miR-124 is a DGCR8-independent, functional microRNA}

To explore the molecular nature of Sindbis-derived miR124 , we infected wild-type murine fibroblasts and compared the RNA from these infections to identical treatments performed in fibroblasts lacking either Dicer, DGCR8, or the IFN-I receptor component IFNAR1 (Fig. $3 \mathrm{~A})$. Following $24 \mathrm{~h}$ of infection, wild-type murine fibroblasts demonstrated robust synthesis of miR-124 specifically from SV124 infections. As previously shown (Fig. 2A), Sindbis-generated miR-124 is dependent on Dicer activity; however, synthesis of Sindbis-derived miR-124 was not dependent on DGCR8, the essential RNA-binding component of the microprocessor. This is in contrast to endogenous miR-93; as in cells lacking Dicer, deletion of DGCR8 results in a complete loss of the endogenous miRNA. Lastly, we determined whether the DGCR8- and Exportin-5-independent generation of Sindbis-derived miR-124 required an antiviral-specific component. For this, we infected cells lacking a functional IFN-I receptor. These cells, similar to loss of DGCR8 or Exportin-5, demonstrate robust miR-124 synthesis with no evidence of cross-talk between the observed noncanonical processing and the cell's autonomous antiviral defenses.

To determine whether Sindbis-derived miR-124 was functional, we transfected an artificial construct in which the green fluorescent protein (GFP) included a $3^{\prime}$-UTR with tandem repeats of the reverse complement of miR-124 (GFP_miR-124t), thereby making it susceptible to PTGS activity (Fig. 3B). Transfection of GFP_miR-124t resulted in robust GFP expression in the absence of any other treatment. In contrast, p124 induced PTGS of GFP_miR-
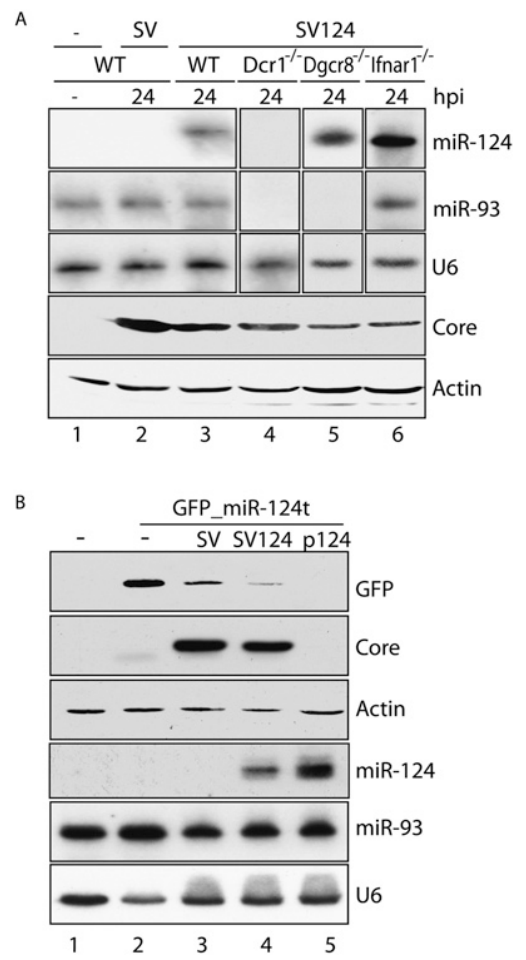

FIGURE 3. (A) Murine embryonic fibroblasts derived from wild-type (WT), Dicer-deficient $\left(\mathrm{Dcrl}^{-1-}\right)$, DGCR8-deficient $\left(\mathrm{Dgcr8}^{-1-}\right)$, or IFN-I-deficient $\left(\right.$ Ifnar $\left.^{-I-}\right)$ mice were mock-treated or infected with SV or SV124 for $24 \mathrm{~h}$ (MOI of 2). (Top three panels) Northern blots probed for miR-124, miR-93, and U6. (Bottom two panels) Western blots for Sindbis virus core protein and actin. (B) Human fibroblasts transfected with a miR-124-targeted GFP plasmid (GFP_miR-124t) were additionally transfected with an miR-124-producing plasmid (p124) or infected with SV or SV124 for $24 \mathrm{~h}$ (MOI of 2). (Top three panels) Western blots for green fluorescent protein (GFP), Sindbis virus core protein, and actin. (Bottom three panels) Northern blots probed for miR-124, miR-93, and U6.

124t to a level below Western blot detection. Furthermore, whereas treatment with SV resulted in a general decrease in host protein synthesis, a common attribute amongst alphaviruses, this effect was significantly enhanced by the production of Sindbis-derived miR-124, suggesting that virus-produced miR-124 was capable of inducing PTGS.

The generation of miR-124, coupled with robust viral replication, suggests that miRNA processing is limited to the subgenomic mRNA transcript rather than directly from the genomic RNA. However, how this pri-miRNA $\sim 500$-nt transcript, which would contain a $5^{\prime}$ cap and a $3^{\prime}$ polyadenylated tail, is processed in the cytoplasm remains unknown. DGCR8 independence and Dicer dependence is reminiscent of previously identified mirtrons (Berezikov et al. 2007; Ruby et al. 2007). Furthermore, the levels of $3^{\prime}$ heterogeneity of Sindbis-derived miR-124 are comparable to that of miR-344, miR-668, and miR-702, three mirtrons previously identified from cells lacking DGCR8 (Babiarz et al. 2008). In contrast, Exportin-5 independence, over-all GC content, and the hairpin end structures of 
Sindbis-derived miR-124 do not conform to the attributes of previously characterized endogenous mirtrons. For this reason, virus-produced mirtron-like products (herein referred to as virtrons) seem to represent a novel cytoplasmic pathway capable of producing functional small RNAs. Future efforts will need to focus on the molecular mechanisms by which virtrons are processed, allowing for PTGS activity.

\section{Virtrons mediate limited Dicer-dependent self-targeting}

As processing of the Sindbis virtron in many ways mimics the antiviral response in invertebrates, we investigated whether Sindbis-derived miR-124 could be generated by Dicer and used in an antiviral capacity in vertebrate cells. While we observed no evidence of attenuation in immortalized human fibroblasts, rapid replication in these cells in response to infections performed at high MOIs may have masked this phenotype. We therefore compared the viral replication properties of SV and SV124 in wild-type (WT), $D c r 1^{-1-}$, and Ifnar $1^{-1-}$ fibroblasts at a low MOI (Fig. 4A). While both SV and SV124 infections amplified to high titers in WT cells by $48 \mathrm{hpi}$, SV124 demonstrated an $\sim 2 \log$ attenuation $(p=0.008)$. This attenuation was not the result of steric hindrance on the RdRp or increased PAMP pro- duction as the levels between SV and SV124 were not significantly different in Dicer knockout cells $(p=0.164)$ but still maintained a $1 \log$ difference in the absence of IFN-I signaling $(p=0.015)$.

To demonstrate that miR-124 could, in fact, be used to target virus directly, we transfected fibroblasts with vector alone or p124 and subsequently mock-treated or infected these cells with SV or SV124 (Fig. 4B). While expression of plasmid-derived miR-124 had no impact on SV core levels, SV124 protein was reduced by 5.8-fold. miR-124 targeting of SV124 likely occurs at the level of the negative strand (-) genome as the mean free energy (mfe) of the miR-124 target on the genome is only $-24.9 \mathrm{kcal} / \mathrm{mol}$ and does not contain a seed sequence $>6 \mathrm{nt}$ (Fig. 4C). This is in contrast to the $(-)$ genome, which has a perfect miR-124 target and an $\mathrm{mfe}$ of $-45.1 \mathrm{kcal} / \mathrm{mol}$.

The emergence and proficiency of PRRs in vertebrates have led to the evolutionary profitable replacement of RNAi-mediated defenses. The processing and functionality of a cytosolic RNA hairpin may, however, suggest that remnants of this system are sill intact, albeit functioning at a diminished level when compared to its invertebrate predecessor. Given that virtrons essentially result in a level of self-targeting, it is unlikely that they represent bona fide viral products. The natural production of virtrons, if in existence, would therefore more likely represent viral by-products of the cell. While this idea is exciting, clearly more extensive research will be needed to determine whether virtrons represent a novel "antique" antiviral pathway and how utilized they are in the cell's antiviral arsenal.

\section{MATERIALS AND METHODS}

B

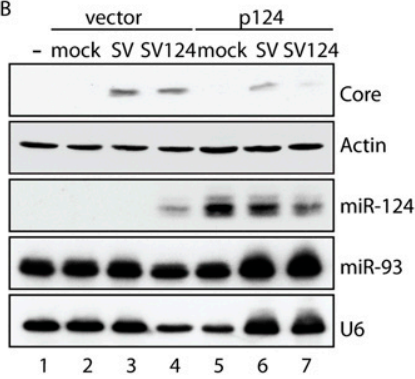

C

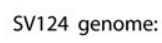

$5^{\prime} \mathrm{C}$ GUGUUCAC A A IIIIIIIIIII IIII

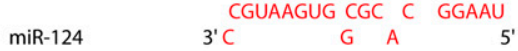
GGCAUUCACCGCGUGCCUUA I I I I I I I I I I I I I I I I I I
CCGUAAGUGGCGCACGGAAU

miR-124 3' CGUAGUG SV124 (-) genome: 5' U

FIGURE 4. (A) Multicycle growth curve of SV and SV124 performed in wild-type murine fibroblasts (WT), or fibroblasts lacking either Dicer $\left(D c r 1^{-1-}\right)$ or a functional IFN-I receptor (Ifnar $1^{-/-}$). Cells were infected at an MOI of 0.1 and plaqued at the indicated time points. $P$-values of the difference between SV and SV124 replication levels in WT, Dcrl ${ }^{-1-}$, and Ifnar $1^{-/-}$at 48 hpi are $0.008,0.164$, and 0.015 , respectively. (B) Human fibroblasts were mocktreated or transfected with vector or miR-124-producing plasmid (p124). Twenty-four hours post-transfection, cells were infected with SV or SV124 (MOI of 2) and harvested 24 hpi. (Top two panels) Western blots for Sindbis virus core protein and actin. (Bottom three panels) Northern blots probed for miR-124, miR-93, and U6. (C) Schematic of miR-124 targeting of the SV124 genome (top) or the SV124 negative-strand genome.

\section{Cell culture and RNA interference}

HEK293, Vero, BHK21, and murine fibroblasts were cultured in DMEM media supplemented with $10 \%$ Fetal Bovine Serum and $1 \%$ penicillin/streptomycin. Dicer-deficient fibroblasts were a kind gift from A. Tarakhovsky (Rockefeller University, NYC) and Donal O'Carrol (EMBL, Monterotondo). DGCR8-deficient fibroblasts were a kind gift from Robert Blelloch (UCSF, CA). RNA interference was performed using pools of scrambled oligonucleotides generated against GFP or human Exportin-5 (Santa Cruz Biotechnologies, sc-45924 and sc-45569). One hundred micromolar RNA oligonucleotide pools were transfected into HEK293 cells in suspension with Lipofectamine 2000 (Invitrogen). Cells were harvested for confirmation of knockdown at 48 and $96 \mathrm{~h}$ post-transfection. 


\section{Virus design, rescue, and infections}

The modified Sindbis viruses described were generated by cloning the miR-124-2 murine locus (chr3:17,695,454-17,696,037) into the Sindbis virus clone TE12Q, a genetically modified strain that contains a duplicate subgenomic mRNA promoter and a unique BstEII cloning site downstream from the structural genes. The primiR-124-2 and the TE12Q constructs have all been described elsewhere (Cheng et al. 1996; Perez et al. 2009; Varble et al. 2010). miR-124 inserts were amplified using previously described primers flanked with BstEII linkers and were cloned directly into the SP6-based DNA plasmid. Viral RNA was subsequently transcribed using the mMessage mMachine SP6 Kit (Ambion) and electroporated into BHK21 cells using AMAXA as per the manufacturer's instructions. Viral infections were performed at the multiplicity of infections (MOIs) specified. Virus was inoculated into indicated cell lines containing serum-free DMEM for $1 \mathrm{~h}$. Inoculum was then aspirated off and replaced with complete medium for the indicated times. Multicycle growth curves were performed in wild-type murine fibroblasts, or fibroblasts lacking functional Dicer or IFNAR1. Briefly, cells were infected with Sindbis virus recombinants at an MOI of 0.1 , and $0.25 \mathrm{~mL}$ of supernatant was removed at the indicated times. Supernatant was plaqued in Vero cells in serial dilutions in triplicate in $1 \%$ methylcellulose. Plaques were counted after $3 \mathrm{~d}$ post-infection. $P$-values were calculated based on a two-tailed $t$-test. Error bars represent the standard deviation $(n=3)$.

\section{Small RNA Northern blot and PTGS analyses}

Small RNA Northern blots and probe labeling were performed as previously described (Pall and Hamilton 2008; Perez et al. 2010). The probes used include anti-miR-124: 5'-TGGCATTCACCGCG TGCCTTAA-3'; anti-miR-93: 5'-CTACCTGCACGAACAGCACT TTG-3'; and anti-U6: 5'-GCCATGCTAATCTTCTCTGTATC-3'. GFP_124t cloning and usage have been described elsewhere (Varble et al. 2010).

\section{Western blot analysis}

Western blots were generated from total protein separated on a $10 \%$ SDS-PAGE gel. Resolved protein was transferred to nitrocellulose (Bio-Rad), blocked for $1 \mathrm{~h}$ with $5 \%$ skim milk at $25^{\circ} \mathrm{C}$, and then incubated with the indicated antibody overnight at $4^{\circ} \mathrm{C}$. The Sindbis core antibody (ATCC, VR-1248AF), Exportin-5 (Santa Cruz Biotechnologies, sc-66885), and GFP (Santa Cruz Biotechnologies, sc-73556) antibodies were used at a concentration of $1 \mu \mathrm{g} / \mathrm{mL}$ in $5 \%$ skim milk. Secondary mouse and rabbit antibodies (GE Healthcare) were used at a 1:5000 dilution for $1 \mathrm{~h}$ at $25^{\circ} \mathrm{C}$. Immobilon Western Chemiluminescent HRP Substrate (Millipore) was used as directed.

\section{miR-124 cloning and sequencing}

miR-124-specific small RNA libraries were generated as previously described (Pfeffer et al. 2005). Briefly, total RNA from indicated samples was isolated using TriZol (Invitrogen) and spiked with radiolabeled size markers (19- and 24-nt oligoribonucleotides: 5' -CGUACGGUUUAAACUUCGA-3' and 5' -CGUACGGCCUAA ACUUCGAAAUGU-3') prior to size fractionation on a $12 \%$ denaturing Tris-urea gel. Adenylated $3^{\prime}$ adapter oligonucleotides
(5'-AppCUGUAGGCACCAUCAAU-3') were ligated onto the isolated small RNA species using T4 RNA ligase 2, truncated (NEB). Following size fractionation of ligated products, $5^{\prime}$ adapter oligonucleotides (5' adapter: 5'-ACACUCUUUCCCUACACG ACGCUCUUCCGAUC-3') were added using T4 RNA ligase (Ambion). The final ligation products were reverse-transcribed with the $3^{\prime}$ adapter primer $5^{\prime}$-ATTGATGGTGCCTACAG-3' using Superscript III (Invitrogen). To PCR-amplify the $5^{\prime}$ ends of miR124 transcripts, a primer pair consisting of a $5^{\prime}$ adapter primer (5'-AATGATACGGCGACCACCGAACACTCTTTCCCTACACG ACG-3') and a reverse miR-124 primer (5'-GGCATTCACCGCG TGCCT- $3^{\prime}$ ) were used. Similarly, the $3^{\prime}$ ends of miR-124 transcripts were amplified using the $3^{\prime}$ adapter primer $\left(5^{\prime}\right.$-ATTGATGGTGCC TACAG- $3^{\prime}$ ) and a forward miR-124 primer (5'-TAAGGCACGCG GTGAATG- $3^{\prime}$ ). PCR products were purified (QIAGEN) and cloned into the commercial pCR-TOPO (Invitrogen) vector following the manufacturer's protocol. Individual colonies were analyzed for differential small RNA processing of $5^{\prime}$ and $3^{\prime}$ ends of mature mmumiR-124-2.

\section{Immunofluorescence}

Cells were fixed on glass coverslips by incubating with $4 \%$ formaldehyde overnight at $4^{\circ} \mathrm{C}$. Following two PBS washes, cells were permeabilized with $0.5 \% \mathrm{NP}-40$ detergent in PBS for $10 \mathrm{~min}$ and immediately washed two additional times. The cells were then blocked with a $0.5 \%$ bovine albumin solution (BSA) in PBS for $30 \mathrm{~min}$ at room temperature. Primary antibody was incubated for $2 \mathrm{~h}$ at room temperature at a 1:500 concentration. The polyclonal Sindbis antibody was purchased from ATCC as Sindbis Ascitic Fluid (VR-1248AF). Following four washes in 0.5\% BSA in PBS, cells were incubated with secondary, Dylight488 (Jackson ImmunoResearch), at 1:750 for $1 \mathrm{~h}$ with Hoechst 33342 dye (Invitrogen) added with 15 min remaining. Following four washes, coverslips were mounted on glass slides with Prolong Gold Antifade (Invitrogen). Images were captured with the Leica TCS SP5 DMI microscope at $60 \times$ magnification.

\section{ACKNOWLEDGMENTS}

We thank R. Blelloch (University of California, San Francisco, CA), M. Heise (University of North Carolina, NC), E. Makeyev (Nanyang Technology University, Singapore), A. Tarakhovsky (Rockefeller University, NYC), D. O'Carrol (EMBL, Monterotondo), T. Maniatis (Columbia University, NY), and B. Brown (MSSM, NY) for reagents and advice associated with this study. A.M.P. is supported by the NYU-MSSM Mechanisms of VirusHost Interactions NIH T32 Training Grant. B.R.T. is supported in part by the Pew Charitable Funds and the U.S. Army Research Office.

Received June 9, 2010; accepted August 13, 2010.

\section{REFERENCES}

Ambros V. 2004. The functions of animal microRNAs. Nature 431: 350-355.

Babiarz JE, Ruby JG, Wang Y, Bartel DP, Blelloch R. 2008. Mouse ES cells express endogenous shRNAs, siRNAs, and other 
Microprocessor-independent, Dicer-dependent small RNAs. Genes Dev 22: 2773-2785.

Bartel DP. 2004. MicroRNAs: Genomics, biogenesis, mechanism, and function. Cell 116: 281-297.

Bartel DP. 2009. MicroRNAs: Target recognition and regulatory functions. Cell 136: 215-233.

Berezikov E, Chung WJ, Willis J, Cuppen E, Lai EC. 2007. Mammalian mirtron genes. Mol Cell 28: 328-336.

Bernstein E, Caudy AA, Hammond SM, Hannon GJ. 2001. Role for a bidentate ribonuclease in the initiation step of RNA interference. Nature 409: 363-366.

Billy E, Brondani V, Zhang H, Muller U, Filipowicz W. 2001. Specific interference with gene expression induced by long, doublestranded RNA in mouse embryonal teratocarcinoma cell lines. Proc Natl Acad Sci 98: 14428-14433.

Cheng EH, Levine B, Boise LH, Thompson CB, Hardwick JM. 1996. Bax-independent inhibition of apoptosis by Bcl-XL. Nature 379: 554-556.

Chi SW, Zang JB, Mele A, Darnell RB. 2009. Argonaute HITS-CLIP decodes microRNA-mRNA interaction maps. Nature 460: 479486.

Garcia-Sastre A, Biron CA. 2006. Type 1 interferons and the virushost relationship: A lesson in detente. Science 312: 879-882.

Hutvagner G, Zamore PD. 2002. A microRNA in a multiple-turnover RNAi enzyme complex. Science 297: 2056-2060.

Iwasaki A, Medzhitov R. 2010. Regulation of adaptive immunity by the innate immune system. Science 327: 291-295.

Ketting RF, Fischer SE, Bernstein E, Sijen T, Hannon GJ, Plasterk RH. 2001. Dicer functions in RNA interference and in synthesis of small RNA involved in developmental timing in C. elegans. Genes Dev 15: 2654-2659.

Khvorova A, Reynolds A, Jayasena SD. 2003. Functional siRNAs and miRNAs exhibit strand bias. Cell 115: 209-216.

Knight SW, Bass BL. 2001. A role for the RNase III enzyme DCR-1 in RNA interference and germ line development in Caenorhabditis elegans. Science 293: 2269-2271.

Lee Y, Ahn C, Han J, Choi H, Kim J, Yim J, Lee J, Provost P, Radmark O, Kim S, et al. 2003. The nuclear RNase III Drosha initiates microRNA processing. Nature 425: 415-419.

MacRae IJ, Zhou K, Doudna JA. 2007. Structural determinants of RNA recognition and cleavage by Dicer. Nat Struct Mol Biol 14: 934-940.

Makeyev EV, Zhang J, Carrasco MA, Maniatis T. 2007. The MicroRNA miR-124 promotes neuronal differentiation by triggering brain-specific alternative pre-mRNA splicing. Mol Cell 27: 435448 .

O'Connell RM, Taganov KD, Boldin MP, Cheng G, Baltimore D. 2007. MicroRNA-155 is induced during the macrophage inflammatory response. Proc Natl Acad Sci 104: 1604-1609.

Okamura K, Hagen JW, Duan H, Tyler DM, Lai EC. 2007. The mirtron pathway generates microRNA-class regulatory RNAs in Drosophila. Cell 130: 89-100.

Pall GS, Hamilton AJ. 2008. Improved Northern blot method for enhanced detection of small RNA. Nat Protoc 3: 1077-1084.
Parameswaran P, Sklan E, Wilkins C, Burgon T, Samuel MA, Lu R, Ansel KM, Heissmeyer V, Einav S, Jackson W, et al. 2010. Six RNA viruses and forty-one hosts: Viral small RNAs and modulation of small RNA repertoires in vertebrate and invertebrate systems. PLoS Pathog 6: e1000764. doi: 10.1371/journal.ppat.1000764.

Pedersen IM, Cheng G, Wieland S, Volinia S, Croce CM, Chisari FV, David M. 2007. Interferon modulation of cellular microRNAs as an antiviral mechanism. Nature 449: 919-922.

Perez JT, Pham AM, Lorini MH, Chua MA, Steel J, tenOever BR. 2009. MicroRNA-mediated species-specific attenuation of influenza A virus. Nat Biotechnol 27: 572-576.

Perez JT, Varble A, Sachidanandam R, Zlatev I, Manoharan M, Garcia-Sastre A, tenOever BR. 2010. Influenza A virus-generated small RNAs regulate the switch from transcription to replication. Proc Natl Acad Sci 107: 11525-11530.

Pfeffer S, Sewer A, Lagos-Quintana M, Sheridan R, Sander C, Grasser FA, van Dyk LF, Ho CK, Shuman S, Chien M, et al. 2005. Identification of microRNAs of the herpesvirus family. Nat Methods 2: 269-276.

Reinhart BJ, Weinstein EG, Rhoades MW, Bartel B, Bartel DP. 2002. MicroRNAs in plants. Genes Dev 16: 1616-1626.

Ruby JG, Jan CH, Bartel DP. 2007. Intronic microRNA precursors that bypass Drosha processing. Nature 448: 83-86.

Schwarz DS, Hutvagner G, Du T, Xu Z, Aronin N, Zamore PD. 2003. Asymmetry in the assembly of the RNAi enzyme complex. Cell 115: 199-208.

Selbach M, Schwanhausser B, Thierfelder N, Fang Z, Khanin R, Rajewsky N. 2008. Widespread changes in protein synthesis induced by microRNAs. Nature 455: 58-63.

tenOever BR. 2009. MicroManipulating viral-based therapeutics. Discov Med 8: 51-54.

Umbach JL, Cullen BR. 2009. The role of RNAi and microRNAs in animal virus replication and antiviral immunity. Genes Dev 23: $1151-1164$.

Varble A, Chua MA, Perez JT, Manicassamy B, Garcia-Sastre A, tenOever BR. 2010. Engineered RNA viral synthesis of microRNAs. Proc Natl Acad Sci 107: 11519-11524.

Wang Y, Baskerville S, Shenoy A, Babiarz JE, Baehner L, Blelloch R. 2008. Embryonic stem cell-specific microRNAs regulate the $G_{1}-S$ transition and promote rapid proliferation. Nat Genet 40: 14781483.

Wang HW, Noland C, Siridechadilok B, Taylor DW, Ma E, Felderer K, Doudna JA, Nogales E. 2009. Structural insights into RNA processing by the human RISC-loading complex. Nat Struct Mol Biol 16: 1148-1153.

Yi R, Qin Y, Macara IG, Cullen BR. 2003. Exportin-5 mediates the nuclear export of pre-microRNAs and short hairpin RNAs. Genes Dev 17: 3011-3016.

Zamore PD, Tuschl T, Sharp PA, Bartel DP. 2000. RNAi: Doublestranded RNA directs the ATP-dependent cleavage of mRNA at 21 to 23 nucleotide intervals. Cell 101: 25-33.

Zipprich JT, Bhattacharyya S, Mathys H, Filipowicz W. 2009. Importance of the C-terminal domain of the human GW182 protein TNRC6C for translational repression. RNA 15: 781-793. 

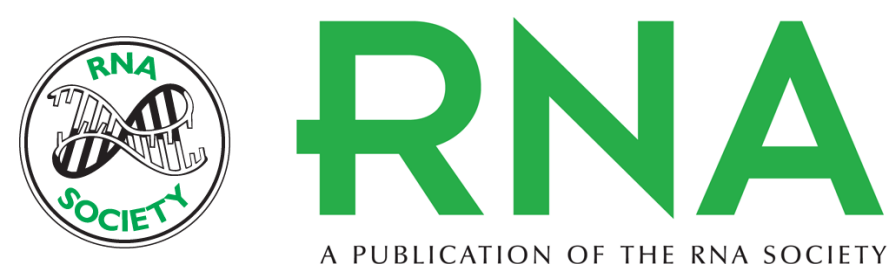

A PUBLICATION OF THE RNA SOCIETY

\section{Noncanonical cytoplasmic processing of viral microRNAs}

Jillian S. Shapiro, Andrew Varble, Alissa M. Pham, et al.

RNA 2010 16: 2068-2074 originally published online September 14, 2010

Access the most recent version at doi:10.1261/rna.2303610

\section{References This article cites 38 articles, 14 of which can be accessed free at: http://rnajournal.cshlp.org/content/16/11/2068.full.html\#ref-list-1}

License

Email Alerting Receive free email alerts when new articles cite this article - sign up in the box at the Service top right corner of the article or click here.

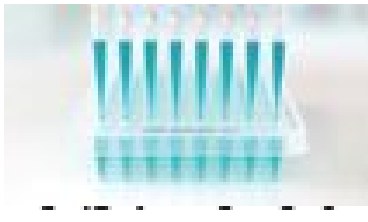

